\title{
Patient-Reported Outcome Measures After
} Hospitalization During the COVID-19 Pandemic: A Survey Among COVID-19 and Non-COVID-19 Patients

\author{
Matan Elkan (1D) \\ Ayana Dvir (D) ${ }^{2}$ \\ Ronit Zaidenstein ${ }^{1,3}$ \\ Maly Keller' \\ Dana Kagansky' \\ Chen Hochman' \\ Ronit Koren ${ }^{1,3}$
}

'Department of Internal Medicine A, Shamir (Assaf Harofeh) Medical Center, Zerifin, Israel; ${ }^{2}$ Intensive Care Unit, Shamir (Assaf Harofeh) Medical Center, Zerifin, Israel; ${ }^{3}$ Sackler Faculty of Medicine, Tel-Aviv University, Tel-Aviv, Israe
Correspondence: Matan Elkan

Department of Internal Medicine A,

Shamir (Assaf Harofeh) Medical Center,

Zerifin, 7030000, Israel

Tel +972-8-977-9242

Fax + 972-8-977-9243

Email matanelkan@gmail.com
Background: Many people recovering from COVID-19 suffer from long-term sequelae. The objective of this study was to assess health-related quality of life (HRQoL) in COVID19 patients several months after discharge.

Methods: We conducted a retrospective cross-sectional case-control study on COVID-19 and non-COVID-19 pneumonia patients admitted to Shamir Medical Center, Israel (03-07/ 2020). In the months following discharge, patients were invited to participate in a survey and fill the RAND-36 questionnaire. Patients' characteristics and comorbidities were extracted from electronic charts.

Results: Among 66 COVID-19 participants, the median age was 58.5 (IQR 49.8-68.3), $56.1 \%$ were female, and $36.4 \%$ were obese. The median length of stay was 7 days (IQR 4 10). Patient-reported outcome measures were reported at a median follow-up of 9-months (IQR 6-9). Pain, general health, vitality, and health change had the lowest scores $(67.5,60$, 57.5 , and 25, respectively). Matching to patients hospitalized with pneumonia due to other pathogens was performed on 42 of the COVID-19 patients. Non-COVID-19 patients were more frequently current or past smokers $(50 \%$ vs $11.9 \%, p<0.01)$ and suffered more often from chronic lung disease $(38.1 \%$ vs $9.5 \%, p=0.01)$. The score for health change was significantly lower in the COVID-19 group (25 vs $50, \mathrm{p}<0.01)$.

Conclusion: Post COVID-19 patients continue to suffer from an assortment of symptoms and perceive a deterioration in their health many months after hospitalization. This emphasizes the importance of prolonged medical follow-up in this population, and the need for additional research to better understand this novel disease's long-term effects.

Keywords: COVID-19, SARS-CoV-2, long COVID, health-related quality of life, patientreported outcome measures

\section{Plain Language Summary}

COVID-19 is a disease caused by the novel SARS-CoV-2 virus, which has rapidly spread over the world, causing significant morbidity and mortality. Patients recovering from this disease may continue suffering from an assortment of symptoms for weeks up to months after acute infection.

In this study, we followed up patients for an average of 9 months after discharge from hospitalization due to COVID-19. Approximately $60 \%$ of the patients still suffered from at least one persistent symptom, most commonly fatigue and myalgia. Additionally, we asked patients to report on their quality of life using a standardized questionnaire. We did not find 
a difference in the quality of life between patients hospitalized with different severity of the disease.

In the second part of our study, we compared patients following hospitalization due to COVID-19 and patients following hospitalization due to pneumonia from other causes. At followup, we did not find a significant difference in the quality-of-life scores between the two groups. Nonetheless, patients recovering from COVID-19 reported that their health was worse than a year ago. In comparison, patients recovering from pneumonia due to other causes do not report such a health change.

Our study emphasizes the long-term impact of COVID-19 and the need for further research regarding its effect on healthrelated quality of life and other patient-reported outcomes.

\section{Introduction}

Over the last year, the global and scientific community has been striving to better understand the different aspects of COVID-19, a novel infectious disease caused by severe acute respiratory syndrome coronavirus 2 (SARS-CoV-2). ${ }^{1}$ During this time, much knowledge has been acquired, and many aspects of this disease have been described, including long-term effects and complications. ${ }^{2,3}$

Many patients continue to suffer post-COVID symptoms, weeks and even months after discharge from hospitalization. Both physical and mental health-related symptoms have been described including breathlessness, fatigue, muscle weakness, anxiety, and depression. ${ }^{4-8}$

In a recent large systematic review of 33 studies, $63.2 \%$ of the patients exhibited one or more post-COVID symptoms 30 days after onset of hospitalization and $45.9 \%$ after 90 days or more. For patients following hospitalization fatigue, dyspnea, and cough were the most common symptoms at 90-day follow-up occurring in 38.5\%, 33.3\%, and $10.4 \%$ of patients, respectively. ${ }^{9}$ Some studies also suggest that symptoms may persist long after recovery and up to 6 months among some patients. ${ }^{10,11}$ For example, Huang et al (2021) have reported that in a mean follow-up of 186 days $76 \%$ of the patients still suffered from at least one symptom, most commonly fatigue or muscle weakness, with a higher risk for patients with severe disease. ${ }^{5}$

As data accumulates regarding the long-term burden from COVID-19, it is important to better understand patient's perception of their health and quality of life, typically known as patient-reported outcome measures (PROM). This can be achieved with validated questionnaires designed to help patients assess their day-to-day physical, and social activities, as well as emotional and mental health. ${ }^{12}$ These measures increase our understanding of disease burden as well as promoting advanced person-centered clinical care and research. ${ }^{13}$

Several studies have described PROMs in patients recovering from COVID-19. For example, in one study Wong et al (2020) report that $76 \%$ of the patients admitted to the hospital with COVID-19 had adverse PROMs 3 months after symptom onset, with one-third of patients reporting at least moderate impairment in major dimensions of quality of life. ${ }^{14}$ In additional studies, healthrelated quality of life (HRQoL) was decreased in the weeks following recovery from acute COVID-19 infection, including decreased mobility and physical functioning, decreased social functioning, anxiety, depression, and pain. $^{4-7,15-18}$

Other pathogens causing pneumonia and hospitalization can adversely affect PROMs and cause a decrease in HRQoL during the months following discharge. This has been previously shown in patients recovering from community-acquired pneumonia even up to 1 year after infection. $^{19-21}$

In this current study, we focus on whether patients continue to suffer from post-COVID symptoms and reduction in HRQoL even beyond 6 months of follow-up. In addition, we consider whether COVID-19 is similar or unique in its impact on HRQoL as compared to patients after hospitalization with non-COVID-associated pneumonia.

\section{Methods}

\section{Patients}

A retrospective cross-sectional case-control study was performed regarding patients 18 years or older, discharged from hospitalization between 1 March and 31 July 2020 from Shamir (Assaf Harofeh) Medical Center (Zerifin, Israel). All patients were admitted to a designated department after SARS-CoV-2 infection was confirmed by RTPCR nasopharyngeal swab. We excluded patients who could not complete the questionnaire (via phone or internet form) due to physical or cognitive impairment. After recruitment, a subset of participants was matched for sex and age to patients hospitalized during the same period due to pneumonia or respiratory infection to an internal medicine department. All patients were tested negative for SARS-CoV-2 during or on admission. In the matched group, patients were excluded if self-reported as being tested positive to SARS-CoV-2 in the follow-up period 
after hospital discharge. The study was approved by the Shamir Medical Center Helsinki Committee for Human Experiments, approval number: 0366-20-ASF.

\section{Data Collection and Definitions}

Data were extracted from electronic medical records including patient demographic information, tobacco smoke addiction, underlying comorbidities, and need for oxygen supplementation. Disease severity was characterized by the highest of a four-category scale, according to guidelines published by the Israeli Ministry of Health. The scale consisted of the following categories: mild disease severity - described as an $\mathrm{O}_{2}$ saturation of $94 \%$ or above without $\mathrm{O} 2$ supplementation, a respiratory rate less than 30 breaths per minute (BPM), and no radiological abnormalities on chest X-ray. Moderate disease severity - signs of COVID-19 pneumonia on chest X-ray and severe disease $\mathrm{O}_{2}$ saturation of $93 \%$ or lower without $\mathrm{O}_{2}$ supplementation or a respiratory rate of $30 \mathrm{BPM}$ or more. Critical disease the need for high flow nasal cannula (HFNC) oxygen supplementation, non-invasive or invasive ventilation.

\section{Patient-Reported Outcomes}

Five to nine months following discharge, patients received a text message offering them to participate in the research study and fill out an online version of the RAND-36 questionnaire. Patients, who were willing to participate but had difficulty filling out the online questionnaire, were contacted and the survey was filled by telephone. All data were collected anonymously.

The RAND 36-Item Health Survey (RAND-36) is a validated, easily administered, and widely used HRQoL survey instrument, which has been previously translated and validated in many languages including Hebrew. ${ }^{22-24}$ It is comprised of 36 items that assess eight health concepts: physical functioning, role limitations caused by physical health problems, role limitations caused by emotional problems, social functioning, emotional well-being, energy/ fatigue, pain, and general health perceptions. Scores for each domain can range from 0 (worst) to 100 (best), higher scores indicate better HRQoL. RAND-36, and the similar license-based SF-36, has been used to evaluate the quality of life in various respiratory and infectious conditions including Middle East Respiratory Syndrome (MERS) and SARS-CoV-1. ${ }^{25,26}$ In addition to the RAND-36 patients were requested to report the persistence of symptoms since discharge.

\section{Statistical Analysis}

All analyses were performed by using SPSS V23.0 (IBM Corp. Armonk, NY, USA). Continuous data are expressed as mean \pm standard deviation (SD) or as the median and interquartile range (IQR) if they were not normally distributed, and categorical data are expressed as frequencies and percentages. Normal distribution was evaluated by histogram and Q-Q plot.

The correlation between continuous and ordinal variables and the outcomes of questionnaires was estimated by using Spearman correlation coefficient. Mann-Whitney test and the Kruskal-Wallis test were used to compare questionnaires' tails between categories.

McNemar's test was used to compare categorical variables between the two matched groups. Paired sample $t$-test and Wilcoxon signed-rank test was applied to compare continuous variables between the two matched groups. P-value was significant if $\alpha<0.05$. All statistical tests were two sided.

\section{Results}

From 1 March to 31 July 2020, 309 patients were discharged from hospitalization due to COVID-19. Sixty-six patients participated in our research (response rate $21.36 \%$ ). Demographic characteristics and comorbidities are shown in Table 1. Age on admission was 58.5 years (49.8-68.3), 56.1\% of the patients being female. More than a third of the patients were obese (36.4\%) with a high mean BMI of $29.1 \mathrm{~kg} / \mathrm{m}^{2}$. After obesity, hypertension was the most frequent comorbidity $(31.8 \%)$, followed by diabetes $(24.2 \%)$, ischemic heart disease $(13.6 \%)$, and chronic lung disease $(12.1 \%)$.

Hospitalization characteristics, length of follow-up, and PROMs are also reported in Table 1. The median length of stay for patients hospitalized for COVID-19 was 7 days (IQR 4-10). Most patients had a mild $(40.9 \%)$ or moderate disease $(30.3 \%)$ not requiring oxygen supplementation in $75.7 \%$ of the cases. Still, $28.8 \%$ of the patients had severe disease with almost a quarter requiring either oxygen supplementation via a nasal prone, mask (19.7\%), HFNC, or noninvasive ventilation $(4.5 \%)$.

PROMs were obtained after a median follow-up of 9-month (IQR 6-9). In the results from the Rand-36 questionnaire emotional role, physical role, social and physical function had the highest scores $(100,87.5,87.5$, and 80 
Table I Demographic Characteristics, Hospitalization and Follow-Up Characteristics, and Patient-Reported Outcomes of Patients Hospitalized Due to COVID-19

\begin{tabular}{|c|c|}
\hline Demographics & $(n=66)$ \\
\hline Age (years), median (IQR) & $58.5(49.8-68.3)$ \\
\hline Female gender (\%) & $37(56.1)$ \\
\hline \multicolumn{2}{|l|}{ Comorbidities } \\
\hline \multicolumn{2}{|l|}{ Smoking } \\
\hline Current (\%) & $3(4.5)$ \\
\hline Past (\%) & $5(7.6)$ \\
\hline Hypertension (\%) & $21(31.8)$ \\
\hline Ischemic heart disease (\%) & $9(13.6)$ \\
\hline Heart failure (\%) & $6(9.1)$ \\
\hline Diabetes mellitus (\%) & $16(24.2)$ \\
\hline Chronic lung disease (\%) & $8(12.1)$ \\
\hline Chronic renal failure (\%) & I (I.5) \\
\hline Obesity (\%) & $24(36.4)$ \\
\hline BMI ( $n=59)$, mean \pm SD & $29.1 \pm 6.5$ \\
\hline \multicolumn{2}{|l|}{ Hospitalization and Follow-Up } \\
\hline Length of stay (days), median (IQR) & $7(4-10)$ \\
\hline \multicolumn{2}{|l|}{ Disease Severity } \\
\hline Mild (\%) & $27(40.9)$ \\
\hline Moderate (\%) & $20(30.3)$ \\
\hline Severe and critical $^{\mathrm{a}}(\%)$ & $19(28.8)$ \\
\hline \multicolumn{2}{|l|}{ Oxygen Status } \\
\hline None (\%) & $50(75.7)$ \\
\hline NP or mask (\%) & $13(19.7)$ \\
\hline HFNC or NIV (\%) & $3(4.6)$ \\
\hline Follow-up (months), median (IQR) & $9(6-9)$ \\
\hline \multicolumn{2}{|c|}{ Additional Hospitalization During Follow-Up } \\
\hline None (\%) & $58(87.9)$ \\
\hline I (\%) & $5(7.6)$ \\
\hline 2 or more (\%) & $3(4.5)$ \\
\hline \multicolumn{2}{|l|}{ Health-Related Quality of Life } \\
\hline Physical functioning, median (IQR) & $80(40-96.2)$ \\
\hline Physical role, median (IQR) & $87.5(25-100)$ \\
\hline Emotional role, median (IQR) & $100(0-100)$ \\
\hline Vitality, median (IQR) & $57.5(30-76.2)$ \\
\hline Emotional well-being, median (IQR) & $74(52-88)$ \\
\hline Social functioning, median (IQR) & $87.5(50-100)$ \\
\hline Pain, median (IQR) & $67.5(24.4-100)$ \\
\hline General health, median (IQR) & $60(35-80)$ \\
\hline Health change, median (IQR) & $25(25-50)$ \\
\hline
\end{tabular}

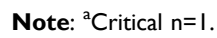

Abbreviations: BMI, body mass index; HFNC, high flow nasal cannula; NIV, noninvasive ventilation; NP, nasal prongs; SD, standard deviation; IQR, interquartile range.

respectively), while emotional well-being, pain, general health, and vitality had lower scores $(74,67.5,60$, and 57.5 respectively) and health change had the lowest score with a median of 25 .

There was no significant association between age or gender and the different HRQoL scores. Overall, patients with comorbidities had lower scores on many of the different categories, some significantly so. In this regard, especially notable is the pain score in which significant differences were found in patients with or without ischemic heart disease ( 22.5 vs $77.5 p=0.006$ ), diabetes ( 33.7 vs $80 p=0.007$ ), lung disease ( 22.5 vs $72.5 p=0.039$ ), and obesity ( 33.7 vs 85 $p=0.017$ ). Considering hospitalization characteristics, no association was found between length of stay and the different scores, although all the different scores were lower by disease severity and need for any oxygen supplementation. However, these differences were not statistically significant. Finally, the length of follow-up was weak but significantly correlated to physical function and emotional well-being $\left(r_{\mathrm{s}}\right.$ $=0.25, p=0.039$, and $r_{\mathrm{s}}=0.28, p=0.022$ respectively). Ingroup associations between patient demographics, patient comorbidities, hospitalization characteristics, and questionnaire scores are shown in Table $\mathrm{S} 1$ and $\underline{\mathrm{S} 2}$.

Many patients complained about the sequela of COVID-19 infection; 57\% of the patients still suffered from at least one symptom and 34\% suffered from two symptoms or more. Most frequently reported was fatigue $(28.8 \%)$, followed by myalgia $(13.6 \%)$, weakness, and shortness of breath (10.6\% each) (Figure 1). Eighty-nine percent of patients with severe disease suffered at least one symptom compared to $65 \%$ and $44 \%$ of the patients with moderate and mild disease severity, respectively.

We matched for age and/or gender a subgroup of 42 COVID-19 patients to patients hospitalized with pneumonia due to other pathogens. A comparison of the groups is shown in Table 2. Non-COVID-19 patients were more frequently current or past smokers $(50 \%$ vs $11.9 \%$, $p=0.003$ ) and suffered more often from chronic lung disease $(38.1 \%$ vs $9.5 \%, p=0.008)$. COVID-19 patients had longer hospitalizations than patients in the non-COVID-19 group ( 8 vs 4 days, $p<0.001$ ) and were also followed up after a shorter period (7.5 vs 9-month, $p<0.001)$. Comparing the different RAND-36 scores, non-COVID -19 patients had lower scores in most categories with a trend towards significance in physical function and role ( 55 vs $80, p=0.070$ and 25 vs $75, p=0.075$ respectively). On the other hand, health change was significantly lower in the COVID-19 group (25 vs 50, $\mathrm{p}=0.004$ ). 


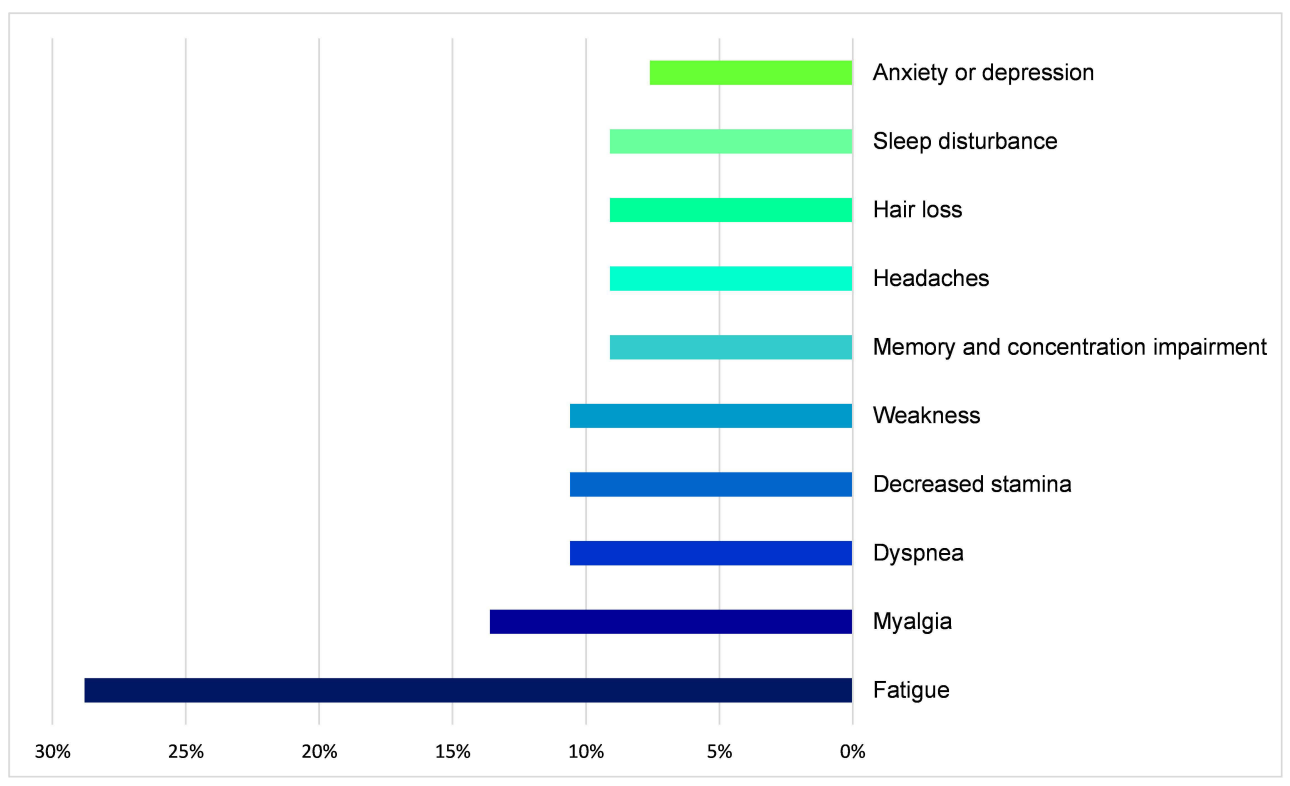

Figure I Frequency of persistent symptoms at follow-up in patients hospitalized due to COVID-19.

\section{Discussion}

It has become increasingly apparent that COVID-19 can have a prolonged impact after acute illness, termed by some "long COVID"27 or "post-acute COVID-19 syndrome". ${ }^{2}$ In this current study, $56 \%$ of the patients still suffered from one or more persistent symptoms at a median follow-up of 9-months. The extent of this problem varies in different studies, depending on the length of follow-up and study population. ${ }^{4-6,10,28,29}$ For example, Logue et al (2021) describe a cohort consisting mainly of outpatients with mild disease in which approximately $30 \%$ of the patients reported persistent symptoms at a median follow-up of 169 days. $^{10}$ While Huang et al (2021) describe a cohort consisting mainly of inpatients with severe disease, where $76 \%$ of the patients reported persistent symptoms in a similar follow-up period. ${ }^{5}$ These results are consistent with our findings, once stratified to disease severity. Forty-five percent of the patients after mild disease, reported at least one post-COVID persistent symptom vs $65 \%$ and $89 \%$ of the patients after moderate and severe disease, respectively.

Fatigue was the most frequent symptom in our cohort reported by $28.8 \%$ of the patients, followed by myalgia in $13.6 \%$ (Figure 1). These findings are consistent with the current literature in which fatigue was the most commonly reported symptom, occurring between $14 \%$ and $71 \%$ of the patients. ${ }^{6,10,28,29}$ The large variability between studies is most probably explained by the different cohort characteristics, comparable to the difference in the total prevalence of persistent symptoms.

HRQoL was also assessed at the time of follow-up. Overall, patients with severe COVID-19 had lower scores in all HRQoL domains, though these findings were not statistically significant, most likely due to the small sample size. Previous studies have reported a decrease in HRQoL across multiple domains with lower scores in patients recovering from severe COVID-19., $, 5,10,14,15,17,18,28$ Simultaneously to persistent symptoms and decreased HRQoL, some studies have also reported residual chest imaging abnormalities and lung diffusion impairment up to 6 months after recovery from acute illness, suggesting one possible explanation for long COVID syndrome. ${ }^{5,15}$

To further understand the long-term effect of COVID19 disease as compared to other respiratory illnesses, we matched a subgroup of our study patients to patients that were hospitalized with non-COVID-19 pneumonia during the same period. Patients with COVID-19 had significantly longer hospitalizations ( 8 vs 4 days), while non-COVID patients were more often smokers (50\% vs $11.9 \%)$ and more frequently had chronic lung disease $(38.1 \%$ vs 9.5\%). Comparing HRQoL, non-COVID patients had lower scores in most domains but with a trend to significance only in physical function and role $(\mathrm{p}=0.070$ and $\mathrm{p}=0.075)$. This difference might be explained due to a larger percentage of patients with comorbid lung disease, both COPD and asthma have been previously shown to 
Table 2 Comparing Demographic Characteristics, Hospitalization and Follow-Up Characteristics, and PROMs Between COVID-19 and Non-COVID-19 Patients

\begin{tabular}{|c|c|c|c|}
\hline Demographics & COVID-19 $(\mathrm{N}=42)$ & Non-COVID $(n=42)$ & $p$ \\
\hline Age (years), median (IQR) & $60(5 I-69.2)$ & 61 (49.7-69) & $0.34 I$ \\
\hline Female gender (\%) & $24(57.1)$ & $25(59.5)$ & $>0.99$ \\
\hline \multicolumn{4}{|l|}{ Comorbidities } \\
\hline Smoking, ever (\%) & $5(11.9)$ & $21(50)$ & 0.003 \\
\hline Hypertension (\%) & $14(33.3)$ & $20(47.6)$ & 0.210 \\
\hline Ischemic heart disease (\%) & $7(16.7)$ & $4(9.5)$ & 0.549 \\
\hline Heart failure (\%) & $4(9.5)$ & $5(11.9)$ & $>0.99$ \\
\hline Diabetes mellitus (\%) & $9(2 \mid .4)$ & $9(2 \mathrm{I} .4)$ & $>0.99$ \\
\hline Chronic lung disease (\%) & $4(9.5)$ & $16(38.1)$ & 0.008 \\
\hline Obesity (\%) & $13(3 \mid)$ & $12(29.3)$ & $>0.99$ \\
\hline BMI, mean \pm SD & $28.2 \pm 5.4$ & $28.4 \pm 4.8$ & 0.836 \\
\hline \multicolumn{4}{|l|}{ Hospitalization and Follow-Up } \\
\hline Length of stay (days), median (IQR) & $8(5-10.2)$ & $4(3-5)$ & $<0.001$ \\
\hline Follow-up (month), median (IQR) & $7.5(6-9)$ & $9(7-10)$ & $<0.001$ \\
\hline Additional hospitalization during follow-up (\%) & $6(14.3)$ & $5(11.9)$ & $>0.99$ \\
\hline \multicolumn{4}{|l|}{ Health-Related Quality of Life } \\
\hline Physical functioning, median (IQR) & $80(43.7-100)$ & $55(8.7-95)$ & 0.070 \\
\hline Physical role, median (IQR) & $75(18.7-100)$ & $25(0-75)$ & 0.075 \\
\hline Emotional role, median (IQR) & $83.3(0-100)$ & $100(0-100)$ & 0.726 \\
\hline Vitality, median (IQR) & $60(28.7-7 \mid .2)$ & $50(23.7-80)$ & 0.614 \\
\hline Emotional well-being, median (IQR) & $76(52-88)$ & $68(47-85)$ & 0.519 \\
\hline Social functioning, median (IQR) & $87.5(46.9-100)$ & $75(37.5-100)$ & 0.348 \\
\hline Pain, median (IQR) & $67.5(24.4-100)$ & $45(11.9-90)$ & 0.201 \\
\hline General health, median (IQR) & $65(38.7-80)$ & $55(37.5-75)$ & 0.247 \\
\hline Health change, median (IQR) & $25(25-50)$ & $50(43.7-75)$ & 0.004 \\
\hline
\end{tabular}

Note: Bold font denotes $\mathrm{p}<0.05$.

Abbreviations: BMI, body mass index; SD, standard deviation; IQR, interquartile range.

decrease HRQoL, especially in severe disease and in patients with frequent exacerbations. ${ }^{30-32}$

In contrast to other domains, the health change score was significantly lower in the group of COVID-19 patients ( 25 vs $50, \mathrm{p}=0.004$ ). This score is constructed by the patient's perception of their change in health relative to a year before, where a score of 50 is equal to no health change and a score of 25 is equal to their health being somewhat worse. This finding may be explained by the high percentage of patients suffering from persistent symptoms at follow-up, causing a reduction in health perception not explained by patient comorbidities.

This study has several limitations including its small sample size, low response rate, and lack of baseline evaluation of participant's HRQoL. Further studies are needed to understand the long-term impact of this novel disease.

\section{Conclusions}

Our study suggests that patients continue to suffer from an assortment of symptoms many months after hospitalization with COVID-19. Fatigue and myalgia were the most common persistent symptoms in $28.8 \%$ and $13.6 \%$ of the patients, respectively. Compared to patients after hospitalization with non-COVID pneumonia, patients recovering from hospitalization with COVID-19 were more likely to report that their health was somewhat worse than the previous year. This emphasizes the importance of pronged medical followup in this population, and the need for additional research to better understand this novel disease's long-term effects.

\section{Abbreviations}

PROM, Patient-reported outcome measures; HRQoL, health-related quality of life; SARS-CoV-2, severe acute 
respiratory syndrome coronavirus 2; BPM, breaths per minute; HFNC, High flow nasal cannula; MERS, Middle East Respiratory Syndrome; SD, standard deviation; IQR, interquartile range; BMI, body mass index; NIV, noninvasive ventilation.

\section{Data Sharing Statement}

Available upon request from the author Dr Matan Elkan matanelkan@gmail.com.

\section{Ethics Approval}

Our study complies with the Declaration of Helsinki and was approved by the Shamir Medical Center Helsinki Committee for Human Experiments, before its initiation. Approval number: 0366-20-ASF.

\section{Consent to Participate}

Informed consent was waived by the local Helsinki Committee for retrospective review of patient records. Written informed consent was waived by the same committee for the questionnaire. The nature of the study was explained to the participants before filling the questionnaire and agreeing by phone or filling the questionnaire by internet form was considered consent to participate in the study.

\section{Consent for Publication}

All authors give their consent for publication.

\section{Disclosure}

The authors have no relevant financial or non-financial conflicts of interests to disclose.

\section{References}

1. Hu B, Guo H, Zhou P, Shi Z-L. Characteristics of SARS-CoV-2 and COVID-19. Nat Rev Microbiol. 2021;19(3):141-154. doi:10.1038/ s41579-020-00459-7

2. Nalbandian A, Sehgal K, Gupta A, et al. Post-acute COVID-19 syndrome. Nat Med. 2021;27(4):601-615. doi:10.1038/s41591-02101283-z

3. SeyedAlinaghi S, Afsahi AM, MohsseniPour M, et al. Late complications of COVID-19; a systematic review of current evidence. Arch Acad Emerg Med. 2021;9(1):e14-e14.

4. Jacobs LG, Gourna Paleoudis E, Lesky-Di Bari D, et al. Persistence of symptoms and quality of life at 35 days after hospitalization for COVID-19 infection. PLoS One. 2020;15(12):e0243882. doi:10.1371/journal.pone. 0243882

5. Huang C, Huang L, Wang Y, et al. 6-month consequences of COVID-19 in patients discharged from hospital: a cohort study. Lancet. 2021;397 (10270):220-232. doi:10.1016/S0140-6736(20)32656-8

6. Garrigues E, Janvier P, Kherabi Y, et al. Post-discharge persistent symptoms and health-related quality of life after hospitalization for COVID-19. $J$ Infect. 2020;81(6):e4-e6. doi:10.1016/j.jinf.20 20.08.029
7. Osikomaiya B, Erinoso O, Wright KO, et al. 'Long COVID': persistent COVID-19 symptoms in survivors managed in Lagos State, Nigeria. BMC Infect Dis. 2021;21(1):304. doi:10.1186/s12879-020-05716-x

8. Xiong Q, Xu M, Li J, et al. Clinical sequelae of COVID-19 survivors in Wuhan, China: a single-centre longitudinal study. Clin Microbiol Infect. 2021;27(1):89-95. doi:10.1016/j.cmi.2020.09.023

9. Fernández-de-las-peñas C, Palacios-Ceña D, Gómez-Mayordomo V, et al. Prevalence of post-COVID-19 symptoms in hospitalized and non-hospitalized COVID-19 survivors: a systematic review and meta-analysis. Eur J Intern Med. 2021. doi:10.1016/j.ejim.2021.06.009

10. Logue JK, Franko NM, McCulloch DJ, et al. Sequelae in adults at 6 months after COVID-19 infection. JAMA Netw Open. 2021;4(2): e210830. doi:10.1001/jamanetworkopen.2021.0830

11. Nasserie T, Hittle M, Goodman SN. Assessment of the frequency and variety of persistent symptoms among patients with COVID-19: a systematic review. JAMA Netw Open. 2021;4(5):e2111417e2111417. doi:10.1001/jamanetworkopen.2021.11417

12. Johnston B, Patrick D, Devji T, et al. Chapter 18: patient-reported outcomes | cochrane training. In: Cochrane Handbook for Systematic Reviews of Interventions Version 6.0. 2019.

13. Williams K, Sansoni J, Darcy M, Grootemaat P, Thompson C. Patient-reported outcome measures. Sydney: Australian Commission on Safety and Quality in Health Care; 2016. Available from: https://www.safetyandquality.gov.au/sites/default/files/ migrated/PROMs-Literature-Review-December-2016.pdf.

14. Wong AW, Shah AS, Johnston JC, Carlsten C, Ryerson CJ. Patientreported outcome measures after COVID-19: a prospective cohort study. Eur Respir J. 2020;56(5):2003276. doi:10.1183/13993003.03276-2020

15. van der $S$, van der Brugge $S$, Talman S, et al. Pulmonary function and health-related quality of life after COVID-19 pneumonia. Respir Med. 2021;176:106272. doi:10.1016/j.rmed.2020.106272.

16. Walle-Hansen MM, Ranhoff AH, Mellingsæter M, Wang-Hansen MS, Myrstad M. Health-related quality of life, functional decline, and long-term mortality in older patients following hospitalisation due to COVID-19. BMC Geriatr. 2021;21(1):199. doi:10.1186/ s12877-021-02140-x

17. Temperoni C, Grieco S, Pasquini Z, et al. Clinical characteristics, management and health related quality of life in young to middle age adults with COVID-19. BMC Infect Dis. 2021;21(1):134. doi:10.1186/s12879-021-05841-1

18. Shah R, Ali FM, Nixon SJ, Ingram JR, Salek SM, Finlay AY. Measuring the impact of COVID-19 on the quality of life of the survivors, partners and family members: a cross-sectional international online survey. BMJ Open. 2021;11(5):e047680. doi:10.1136/ bmjopen-2020-047680

19. Glick HA, Miyazaki T, Hirano K, et al. One-year quality of life postpneumonia diagnosis in Japanese adults. Clin Infect Dis. 2020;73:283. doi:10.1093/cid/ciaa595

20. Mangen M-J-J, Huijts SM, Bonten MJM, de Wit GA. The impact of community-acquired pneumonia on the health-related quality-of-life in elderly. BMC Infect Dis. 2017;17(1):208. doi:10.1186/s12879-0172302-3

21. Andrade LF, Saba G, Ricard J-D, et al. Health related quality of life in patients with community-acquired pneumococcal pneumonia in France. Health Qual Life Outcomes. 2018;16(1):28. doi:10.1186/ s12955-018-0854-6

22. Hays RD, Morales LS. The RAND-36 measure of health-related quality of life. Ann Med. 2001;33(5):350-357. doi:10.3109/ 07853890109002089

23. Hays RD, Sherbourne CD, Mazel RM. The rand 36-item health survey 1.0. Health Econ. 1993;2(3):217-227. doi:10.1002/ hec. 4730020305

24. Lewin-Epstein N, Sagiv-Schifter T, Shabtai EL, Shmueli A. Validation of the 36-item short-form health survey (Hebrew version) in the adult population of Israel. Med Care. 1998;36(9):1361-1370. doi:10.1097/00005650-199809000-00008 
25. Ngai JC, Ko FW, Ng SS, To K-W, Tong M, Hui DS. The long-term impact of severe acute respiratory syndrome on pulmonary function, exercise capacity and health status. Respirology. 2010;15(3):543-550. doi:10.1111/j.1440-1843.2010.01720.x

26. Batawi S, Tarazan N, Al-Raddadi R, et al. Quality of life reported by survivors after hospitalization for Middle East respiratory syndrome (MERS). Health Qual Life Outcomes. 2019;17(1):101. doi:10.1186/ s12955-019-1165-2

27. Mahase E. Covid-19: what do we know about "long covid"? BMJ. 2020;370:m2815. doi:10.1136/bmj.m2815

28. Carfì A, Bernabei R, Landi F. Persistent symptoms in patients after acute COVID-19. JAMA. 2020;324(6):603. doi:10.1001/jama.20 20.12603
29. Tenforde MW, Billig Rose E, Lindsell CJ, et al. Characteristics of adult outpatients and inpatients with COVID-19-11 academic medical centers, United States, March-May 2020. MMWR Morb Mortal Wkly Rep. 2020;69(26):841-846. doi:10.15585/mmwr.mm6926e3

30. Esteban C, Quintana JM, Moraza J, et al. Impact of hospitalisations for exacerbations of COPD on health-related quality of life. Respir Med. 2009;103(8):1201-1208. doi:10.1016/j.rmed.2009.02.002

31. Ståhl E, Lindberg A, Jansson S-A, et al. Health-related quality of life is related to COPD disease severity. Health Qual Life Outcomes. 2005;3(1):56. doi:10.1186/1477-7525-3-56

32. Adams R, Wakefield M, Wilson D, et al. Quality of life in asthma: a comparison of community and hospital asthma patients. J Asthma. 2001;38(3):205-214. doi:10.1081/JAS-100000107

\section{Publish your work in this journal}

The International Journal of General Medicine is an international, peer-reviewed open-access journal that focuses on general and internal medicine, pathogenesis, epidemiology, diagnosis, monitoring and treatment protocols. The journal is characterized by the rapid reporting of reviews, original research and clinical studies
International Journal of General MedicineDovepress

across all disease areas. The manuscript management system is completely online and includes a very quick and fair peer-review system, which is all easy to use. Visit http://www.dovepress.com/ testimonials.php to read real quotes from published authors. 